\title{
Nonequilibrium dynamics of the phonon gas in ultrafast-excited antimony
}

\author{
Sergej Krylow, ${ }^{1}$ Eeuwe S. Zijlstra, ${ }^{1}$ Fairoja Cheenicode Kabeer, ${ }^{2}$ Tobias Zier, ${ }^{1}$ Bernd Bauerhenne, ${ }^{1}$ and Martin E. Garcia ${ }^{1}$ \\ ${ }^{1}$ Theoretical Physics and Center for Interdisciplinary Nanostructure Science and Technology (CINSAT), University of Kassel, \\ Heinrich-Plett-Str. 40, 34132 Kassel, Germany \\ ${ }^{2}$ Fritz-Haber-Institut der Max-Planck-Gesellschaft, Faradayweg 4-6, D-14195 Berlin, Germany
}

(Received 21 March 2017; published 19 December 2017)

\begin{abstract}
The ultrafast relaxation dynamics of a nonequilibrium phonon gas towards thermal equilibrium involves manybody collisions that cannot be properly described by perturbative approaches. Here, we develop a nonperturbative method to elucidate the microscopic mechanisms underlying the decay of laser-excited coherent phonons in the presence of electron-hole pairs, which so far are not fully understood. Our theory relies on $a b$ initio molecular dynamics simulations on laser-excited potential-energy surfaces. Those simulations are compared with runs in which the laser-excited coherent phonon is artificially deoccupied. We apply this method to antimony and show that the decay of the $A_{1 g}$ phonon mode at low laser fluences can be accounted mainly to three-body down-conversion processes of an $A_{1 g}$ phonon into acoustic phonons. For higher excitation strengths, however, we see a crossover to a four-phonon process, in which two $A_{1 g}$ phonons decay into two optical phonons.
\end{abstract}

DOI: 10.1103/PhysRevMaterials.1.073601

\section{INTRODUCTION}

An accurate microscopic description of the ultrafast dynamics of a nonequilibrium phonon gas is of fundamental importance for understanding energy-redistribution processes at the subpicosecond scale. However, the first stages in the evolution of a nonthermal phonon gas towards a common lattice temperature in the presence of hot electrons are, to a large extent, still unknown. A typical nonthermal phonon gas can be generated, for instance, by an ultrashort laser pulse through the excitation of a coherent optical phonon $[1,2]$ and the simultaneous softening or hardening of covalent bonds [3-6]. While the excitation mechanisms of coherent phonons have been intensively studied and are well understood [7-13], the decay channels through phonon-phonon interactions in laser-excited solids have not yet been fully elucidated. Previous studies included equilibrium and perturbative approaches based on a damped-oscillator equation and on Fermi's golden rule $[14,15]$. However, a nonperturbative, nonequilibrium description of the involved equilibration processes is lacking. In this paper, we develop and apply a method to describe and analyze the dynamics of a coherent phonon on an $a b$ initio determined potential-energy surface (PES). The PES is, contrary to usual methods, not restricted on its functional form and, thus, includes all possible phonon-phonon interactions. Therefore, no initial analysis of the possible phonon-phonon interactions, contrary to previous studies, is needed for our calculations.

Our approach is based on two types of ab initio molecular dynamics (MD) simulations. One type includes the full structural dynamics of the laser-excited solid, termed full runs. For the other type, we artificially deoccupy the coherent phonon without altering any other phonon mode and without changing the laser-excited PES. These additional " $A_{1 g}$-empty" runs describe the dynamics of the nonequilibrium phonon gas in the absence of the coherent phonon. By comparing the full runs with the $A_{1 g}$-empty runs and using general physical arguments, we are able to unravel the decay channels of the nonequilibrium phonon gas. Here, we show the applicability of the method to the example of $\mathrm{Sb}$, which exhibits both laser-induced bond-softening and coherently excitable phonon modes [16,17].

The rest of the paper is organized as follows. In Sec. II A, we state details regarding our simulations. In Sec. II B, we derive the formalism to calculate the phonon occupation number in the harmonic approximation, followed by the description of the generation of the $A_{1 g}$-empty runs in Sec. II C. The analysis of the generation mechanism and the decay of the coherent $A_{1 g}$ phonon, visualized by the Peierls parameter, is discussed in Sec. III A. Then, we describe the different contributions to the dynamics of the phonon gas in Sec. III B. The decay channels of the coherent $A_{1 g}$ phonon mode are discussed in Sec. III C. We conclude our results in Sec. IV.

\section{THEORY}

\section{A. Molecular dynamics simulations of laser-excited antimony}

Our simulations were performed using our in-house Code for Highly excIted Valence Electron Systems (CHIVES), which is two orders of magnitude faster than other state-of-the-art density-functional-theory (DFT) codes for the same accuracy $[18,19]$. In CHIVES, the equations of motion for the atoms are integrated under the influence of a laser-excited PES, which is computed by solving the electronic problem in the local density approximation [20]. CHIVES uses localized electronic basis functions and pseudopotentials [21-23]. We considered a supercell containing $N=576 \mathrm{Sb}$ atoms, which is made up of 48 orthorhombic unit cells, each having 12 atoms. We applied periodic boundary conditions and used a $2 \times 2 \times 2 \mathbf{k}$ grid. We thermalized the system to room temperature [24]. Once the lattice acquired a homogeneous room temperature, we stored the positions and velocities of the atoms every $2 \mathrm{ps}$ in order to generate the initial configurations for the MD runs describing the response of $\mathrm{Sb}$ to the laser pulse. In this way, we generated 80 initial conditions for the same number of MD runs, which were used for the considered laser fluences; see below. Note that this number of simulations was necessary to ensure the statistical relevance of our results. In all MD runs, a time step of 5 fs was used, which corresponds to $\approx 1 / 40$ of the highest 


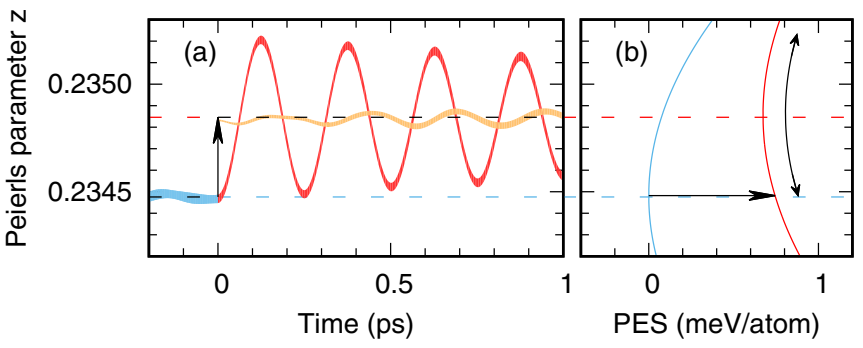

FIG. 1. (a) Oscillation of the Peierls parameter $z(t)$ before and after excitation (at $0 \mathrm{ps}$ ) at an electronic temperature of $1500 \mathrm{~K}$ and averaged over 80 runs. The full runs are shown in red and the $A_{1 g^{-}}$ empty ones are shown in orange. (b) Calculated PES along the $A_{1 g}$ phonon mode before (cyan) and after (red) laser excitation. The offset between the ground and excited state is arbitrarily chosen. (See [25] for details.)

phonon period (see Fig. 2), to guarantee a correct description of the phonon dynamics.

The femtosecond-laser excitation was modeled by changing the electronic temperature from the initial room temperature to an electronic temperature ranging from 1000 to $3500 \mathrm{~K}$. This changes the occupation of the electronic states via a Fermi-Dirac distribution. In this way, the PES is modified along all phonon modes, where a higher electronic temperature corresponds to a higher excitation density. In particular, the PES becomes softened and is shifted in the direction of the $A_{1 g}$ phonon. As a consequence, a coherent phonon is excited displacively [see Fig. 1(b) ]. The $A_{1 g}$ phonon can be tracked by the Peierls parameter $z(t)$, which is a dimensionless variable defined by

$$
|\boldsymbol{d}|=|(0,0,2 z(t))| c,
$$

where $|\boldsymbol{d}|$ is the distance between the two basis atoms in the primitive cell and $c=11.2220 \AA$ is our lattice parameter in the $x_{3}$ direction.

Although our approach constitutes a generalization of the Born-Oppenheimer approximation, it does not include incoherent electron-phonon coupling. We discuss the implications of this in Sec. III A. It is important to point out that the implementation of incoherent electron-phonon scattering at a microscopic level into ab initio MD simulations is still an open problem.

\section{B. Phonon occupation numbers}

In order to analyze the decay channels in $\mathrm{Sb}$, it is convenient to calculate the phonon occupations in each phonon mode. The calculation of the phonon occupation numbers is performed as follows. We expand the ab initio PES obtained from CHIVES up to second order in the atomic displacements (harmonic approximation). This allows us to write the harmonic Hamiltonian $H\left(T_{e}\right)$ as a sum over all $3 N$ phonon modes,

$$
H\left(T_{e}\right)=\frac{1}{2} \sum_{j=1}^{3 N}\left[\bar{p}_{j}^{2}(t)+\omega_{j}^{2}\left(T_{e}\right) \bar{u}_{j}^{2}(t)\right],
$$

with variables $\bar{p}_{j}$ and $\bar{u}_{j}$ defined by

$$
\begin{gathered}
\bar{p}_{j}=\frac{\boldsymbol{e}_{j} \boldsymbol{p}(t)}{\sqrt{M}}, \\
\bar{u}_{j}=\sqrt{M} \boldsymbol{e}_{j} \boldsymbol{u}(t) .
\end{gathered}
$$

The parameter $\omega_{j}\left(T_{e}\right)$ is the $j$ th eigenfrequency of the laserexcited PES with the corresponding eigenvector $\boldsymbol{e}_{j}$, which are calculated by diagonalizing the dynamical matrix, and $M$ is the mass of an antimony atom. The vector $\boldsymbol{p}$ contains the impulses and $\boldsymbol{u}$ the displacements out of the ideal positions at the particular electronic temperature. Except for the Peierls parameter $z_{0}\left(T_{e}\right)$, which is set to the average value obtained in the full runs after laser excitation, the ideal positions are defined by symmetry. Now one can perform a change of variables and define the coefficients

$$
\begin{aligned}
& b_{j}(t)=\sqrt{\frac{\omega_{j}}{2 \hbar}} \bar{u}_{j}(t)+i \sqrt{\frac{1}{2 \hbar \omega_{j}}} \bar{p}_{j}(t), \\
& b_{j}^{*}(t)=\sqrt{\frac{\omega_{j}}{2 \hbar}} \bar{u}_{j}(t)-i \sqrt{\frac{1}{2 \hbar \omega_{j}}} \bar{p}_{j}(t),
\end{aligned}
$$

from which we can calculate the phonon occupation number of the $j$ th phonon by

$$
n_{j}(t)=b_{j}^{*}(t) b_{j}(t)
$$

or, alternatively, the energy by

$$
E_{j}(t)=\hbar \omega_{j} n_{j}(t) .
$$

Note that since we treat the phonons classically, the energy of one particular phonon can be zero. Furthermore, we always average over 80 runs to obtain the time evolution of the phonon occupation numbers or the energy of a particular phonon mode.

\section{C. $A_{1 g}$-empty runs}

As we already mentioned, we perform additional types of runs, which we will refer to as $A_{1 g}$-empty runs. In those modes, we artifically deoccupy the $A_{1 g}$ phonon mode directly after laser excitation. To calculate the $A_{1 g}$-empty runs, we project the momenta and displacements, at time zero, onto the eigenvector of the $A_{1 g}$ phonon mode by Eqs. (3) and (4) for each run. The new positions $r^{\text {empty }}$ and impulses $p^{\text {empty }}$ are calculated by

$$
\boldsymbol{p}^{\mathrm{empty}}=\boldsymbol{p}-\bar{p}_{A_{1 g}}\left(\boldsymbol{e}_{A_{1 g}} \cdot \sqrt{M}\right)
$$

and

$$
\boldsymbol{r}^{\mathrm{empty}}=\boldsymbol{r}_{0}-\bar{u}_{A_{1 g}}\left(\boldsymbol{e}_{A_{1 g}} / \sqrt{M}\right) .
$$

In this way, we only alter the phonon occupation number of the $A_{1 g}$ phonon, which would become zero if no anharmonicities would be present.

\section{RESULTS}

\section{A. Time evolution of the Peierls parameter}

Figure 1(a) shows the time evolution of the Peierls parameter, defined in Eq. (1), before (cyan) and after (red) laser excitation with an electronic temperature of $1500 \mathrm{~K}$. In order to compute the lifetime of the coherent $A_{1 g}$ phonon from our 
simulations, we fitted $z(t)$, obtained from the full runs, with an exponentially decaying function of the form

$$
z_{\mathrm{fit}}(t)=A \cos \left(2 \pi v_{A_{1 g}} t\right) \exp \left(-\frac{t}{\tau}\right)+z_{0}
$$

for the first 4 ps after laser excitation. In Eq. (11), $A=$ $-3.79 \times 10^{-4}$ is the amplitude, $v_{A_{1 g}}=3.99 \mathrm{THz}$ is the frequency, $\tau=3.25 \mathrm{ps}$ is the decay time, and $z_{0}=0.234846$ is the new equilibrium value for the Peierls parameter in the laser-excited PES. The parameters for the other electronic temperatures are given in Table I. One can see that a higher electronic temperature, which corresponds to a higher laser fluence, leads to a bigger amplitude and a stronger softening of the $A_{1 g}$ phonon mode. The magnitude of the calculated softening was also experimentally observed in Refs. [17,26] for laser pulses in the $\mathrm{mJ} / \mathrm{cm}^{2}$ regime. In particular, in Ref. [26], an electronic temperature of $2000 \mathrm{~K}$ was used to simulate an excitation with a laser pulse of fluence $2.6 \mathrm{~mJ} / \mathrm{cm}^{2}$. Our calculated decay times, shown in Table I, are approximately 3 ps for all considered electronic temperatures. This contradicts the fluence dependence of the decay time observed in a recent experiment [17]. A higher fluence leads to a bigger amplitude of the coherent $A_{1 g}$ phonon, which is included in our simulations, but presumably also to an additional increase of the lattice temperature due to incoherent electron-phonon heating. The increase of the lattice temperature and the corresponding decrease of the electronic temperature were theoretically calculated using a thermodynamical model in Refs. [27] and [28] for the parent compound bismuth. From Ref. [29], it is known that the decay time of the coherently excited $A_{1 g}$ phonon mode in antimony depends on the lattice temperature. In our simulations, lattice temperature remains at approximately the initialization temperature of $300 \mathrm{~K}$ during at least 1 ps after the action of the laser pulse. This could be the reason why our determined decay times are similar to those measured by pump-probe experiments for low fluences and room temperature in Ref. [7] (2.9 ps) and in Ref. [29] $(\approx 2.94 \mathrm{ps})$. The fact that a low-fluence laser excitation was used in Ref. [26] could also explain why the time evolution of Bragg-peak intensities was well reproduced within the first picosecond after laser excitation. Note that the error derived by fitting Eq. (11) does not include the effects from incoherent

TABLE I. Parameters of the damped-oscillator model of the coherent phonon for different electronic temperatures fitted to the $a b$ initio simulation using Eq. (11). See text for explanation of the parameters. The fitting error in $A$ is 0.01 , in $v$ is $0.0004 \mathrm{THz}$, in $\tau$ is $0.04 \mathrm{ps}$, and in $z_{0}$ is $3 \times 10^{-6}$.

\begin{tabular}{lcccc}
\hline \hline $\begin{array}{l}\text { Electronic temperature } \\
(\mathrm{K})\end{array}$ & $A$ & $\begin{array}{c}v \\
(\mathrm{THz})\end{array}$ & $\begin{array}{c}\tau \\
(\mathrm{ps})\end{array}$ & $z_{0}$ \\
\hline 300 & & 4.50 & & \\
1000 & -0.14 & 4.1350 & 2.69 & 0.234607 \\
1500 & -0.38 & 3.9928 & 3.25 & 0.234846 \\
2000 & -0.74 & 3.8297 & 3.09 & 0.235196 \\
2500 & -1.24 & 3.6207 & 2.79 & 0.235676 \\
3000 & -1.89 & 3.3711 & 2.77 & 0.236314 \\
3500 & -2.75 & 3.0694 & 3.08 & 0.237226 \\
\hline \hline
\end{tabular}

electron-phonon coupling and should therefore be larger. The decrease of the decay time $\tau$ for increasing lattice temperature, observed in experiments, is probably due to the increase in the occupations of the phonons involved in the relevant decay channels, which in turn increases the rate of decay of the coherent phonon. It is important to point out that the absence of lattice heating due to incoherent electron-phonon coupling in our calculations does not affect at all our calculated decay channels since they are determined by the phonon-phonon interactions, which we take into account exactly for the given PES.

The dynamics of the Peierls parameter averaged over the $A_{1 g}$-empty runs is shown in orange in Fig. 1(a). One can see that the time-dependent Peierls parameter $z(t)$ still exhibits coherent oscillations of low amplitude, in spite of the deoccupation of the $A_{1 g}$ phonon. We attribute these oscillations to anharmonic effects.

\section{B. Bond softening and periodic modulations}

By analyzing only the phonon occupation numbers obtained from the full runs, it is not possible to extract the decay channels of the coherent phonon mode. This is because our computed PES does include, apart from all phononphonon interactions, other laser-induced effects which affect the dynamics of the phonons and are superimposed to the decay of the $A_{1 g}$ phonon. In particular, we were able to extract two additional aspects, which have to be considered in order to analyze the mechanisms leading to the decay of the coherent $A_{1 g}$ phonon. The first one is the laser-induced bond softening, which manifests itself in a change of the potential energy and an ensuing shift of the phonon frequencies [see Fig. 2(a)]. Another aspect is a periodic modulation of the phonon occupation numbers with a period equal to the softened $A_{1 g}$ phonon. Laser-induced bond softening of phonon
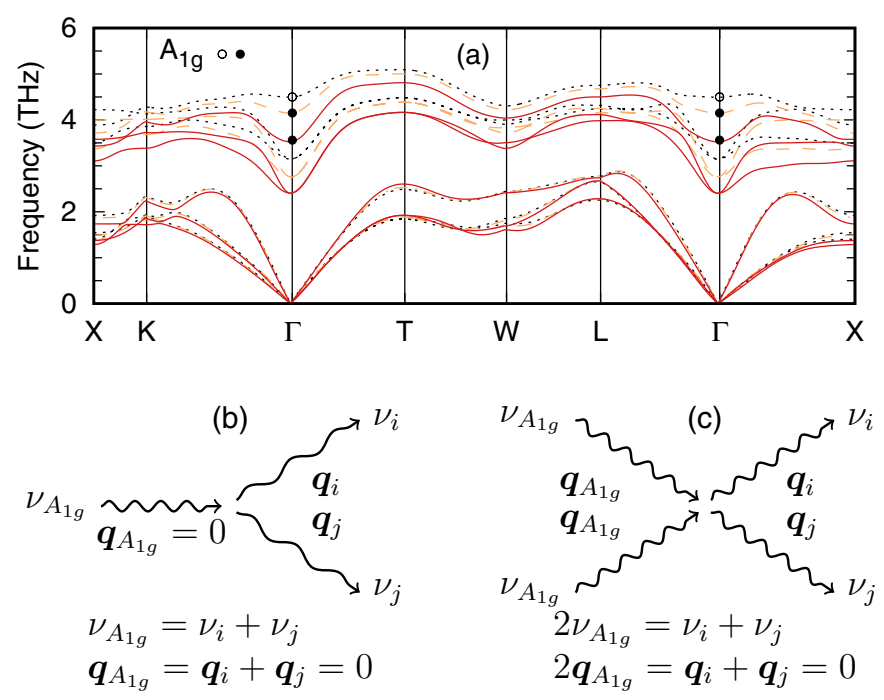

FIG. 2. (a) Phonon-dispersion relation of $\mathrm{Sb}$ at an electronic temperature of $300 \mathrm{~K}$ (black dotted lines), for the excited case with an electronic temperature of $1500 \mathrm{~K}$ (orange dashed lines) and $3000 \mathrm{~K}$ (red solid lines). (b),(c) Diagrams of the proposed third- and fourth-order scattering processes governing the decay at moderateand high-excitation densities. 


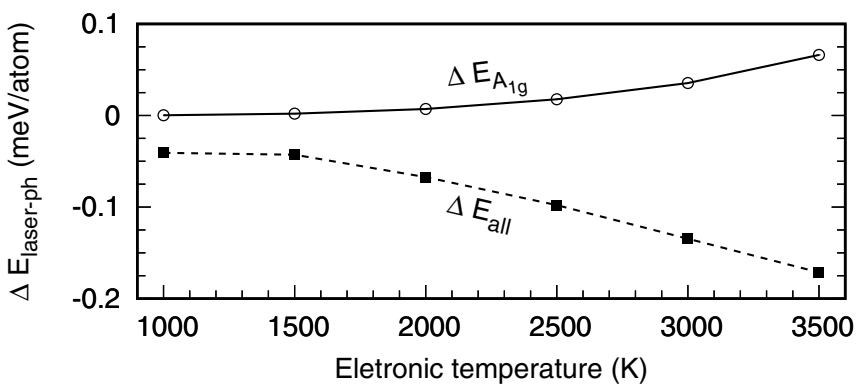

FIG. 3. Shift of the energy at time $t=0$ for the $A_{1 g}$ phonon (solid line) and for all other phonons (dashed line).

modes was already reported in Refs. [30,31] for bismuth, which behaves, in many aspects, similarly to antimony. A periodic modulation of the signal with the same frequency as a previously excited coherent phonon has been observed for carbon nanotubes [32].

In particular, the bond softening leads to a significant nonequilibrium within the laser-excited phonon gas. This can be quantified by calculating the shift of the energy $\Delta E_{\text {laser-ph }}$ due the laser excitation for the $A_{1 g}$ phonon $\left(\Delta E_{A_{1 g}}\right)$ and all other phonon modes $\left(\Delta E_{\text {all }}\right)$ by

$$
\Delta E_{A_{1 g}}=\left(E_{A_{1 g}}^{\mathrm{exc}}-E_{A_{1 g}}^{\mathrm{initial}}\right) / N
$$

and

$$
\Delta E_{\mathrm{all}}=\sum_{j=1}^{3 N}\left(E_{j}^{\mathrm{exc}}-E_{j}^{\mathrm{initial}}\right) / N, j \neq A_{1 g},
$$

respectively. Here, $E_{j}^{\text {initial }}$ and $E_{j}^{\text {exc }}$ are the energies calculated by Eq. (8), which means, in the harmonic approximation, before and directly after $(t=0)$ laser excitation. Figure 3 shows $\Delta E_{A_{1 g}}$ (solid line) and $\Delta E_{\text {all }}$ (dashed line) for all analyzed electronic temperatures. $\Delta E_{\text {all }}$ is entirely due to bond softening and $\Delta E_{A_{1},}$ is mainly due to the shift of the PES after laser excitation. Note that the $A_{1 g}$ phonon also exhibits bond softening, but this effect is small compared to the shift of the PES due to the laser excitation. One can see that the absolute value of the energy shift $\Delta E_{\text {all }}$ is larger than $\Delta E_{A_{1 g}}$ for every analyzed electronic temperature, so that a significant dynamics of the system can be expected due to the bond softening. In fact, we have found two contributions of bond softening to the dynamics of the system in antimony. The first one is related to the fact that the bond softening does not alter the kinetic energy, creating an unbalance between the potential and kinetic energy of the softened phonon. This leads to the well-known laser-induced squeezing [33]. The second contribution of bond softening is a phonon-phonon equilibration process, which occurs because the phonons do not have the same average energy after laser excitation. In particular, one can see an energy transfer from the acoustic phonons, which are less softened, to the optical phonon modes.

\section{Decay channels in Sb}

Since the full and the $A_{1 g}$-empty runs show the same response to bond softening, the main difference will lie in the occupancy redistribution due to the presence of the $A_{1 g}$ phonon. To extract the redistribution processes, we computed the phonon occupations in the $A_{1 g}$-empty runs and subtract them from those of the full runs. Then, and in order to obtain meaningful results, we performed time averages over the $n$th period of the coherent phonon for each electronic temperature. Furthermore, the analysis of the occupancy redistribution for every single phonon mode, out of the 1728 possible ones, is not meaningful. Therefore, we considered occupancy changes in blocks containing 96 consecutive phonon modes, ordered by their phonon frequency and excluding the $A_{1 g}$ phonon.

Below we discuss just the case of an electronic temperature of $1500 \mathrm{~K}$ and then extend the analysis of the decay channels to an electronic temperature of $3000 \mathrm{~K}$. Figures 4(b)-4(e) show the time-averaged changes in phonon occupancies for an
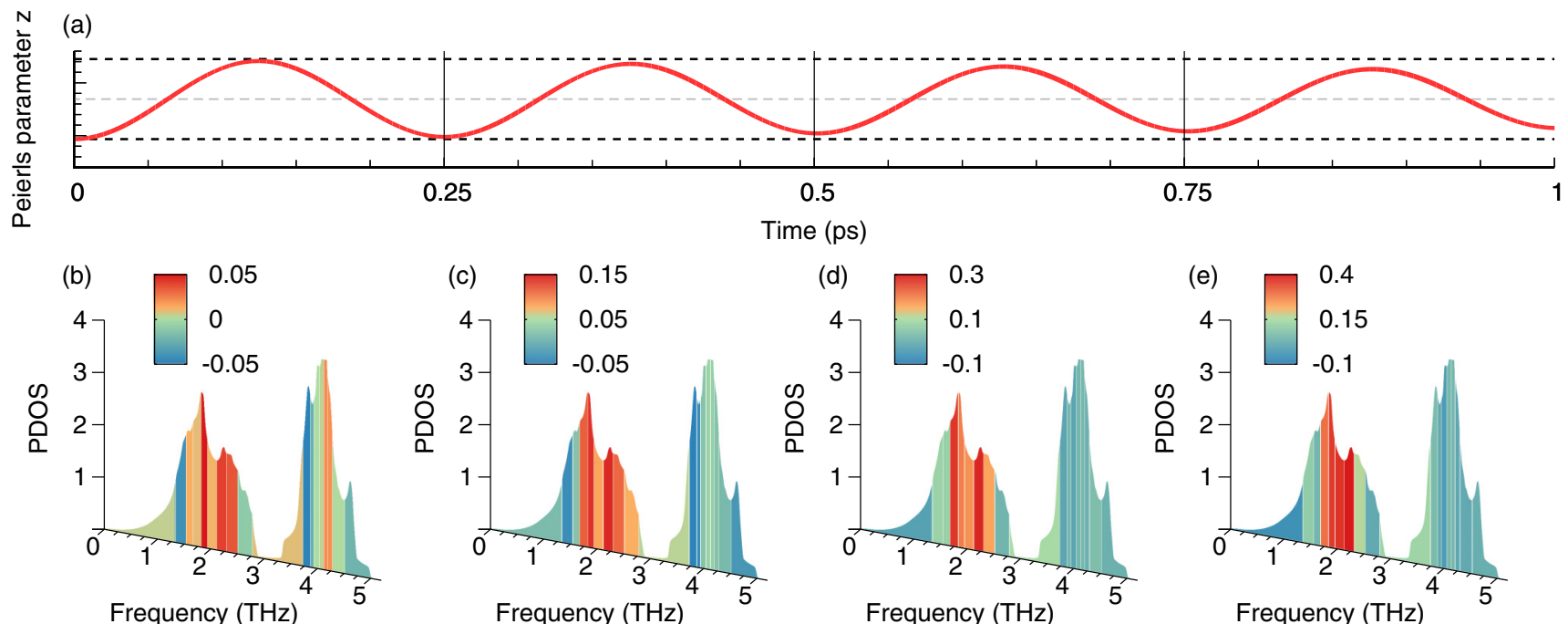

FIG. 4. (a) Oscillation of the Peierls parameter of the $A_{1 g}$ phonon (average from 80 full runs). The tics on the $y$ axis are the same as in Fig. 1(a). (b)-(e) Phonon density of states (PDOS) for an electronic temperature of $1500 \mathrm{~K}$. The colored stripes show the difference in the phonon occupation numbers between the full and $A_{1 g}$-empty runs. These are summed over 96 phonon modes and averaged over a period of the coherent phonon. The average over the period of the coherent phonon is done, from left to right, for the first four periods. 
electronic temperature of $1500 \mathrm{~K}$, superimposed to the phonon density of states (PDOS), as colored stripes corresponding to 96 phonon modes each. The dynamics of the Peierls parameter in Fig. 4(a) indicates the periods of the coherent phonon mode over which time averaging was performed. In Figs. 4(b)-4(e), one can see that mostly phonon occupancies within the frequency range of around $2 \mathrm{THz}$, which is approximately one-half of the $A_{1 g}$ phonon frequency $v_{A_{1 g}}$, increase in time. We point out that the aforementioned increase of the phonon occupancies becomes more pronounced for later periods. For longer times, first, the coherent phonon has transferred more energy to the other phonons and, second, decay processes for which the energy conservation is well defined become dominant. Since the phonon-phonon interactions reflect scattering between waves, one can use the time-frequency uncertainty principle from Fourier analysis to study when collisions are completed. Thus, for $250 \mathrm{fs}$ after laser excitation (this means, during the first $A_{1 g}$ period), the uncertainty in frequency is still as large as $2 \mathrm{THz}$. After two, three, and four $A_{1 g}$ periods, the uncertainty in frequency becomes $1,0.6$, and 0.5 $\mathrm{THz}$, respectively. This qualitative estimate explains the results shown in Figs. 4(b)-4(e).

To understand which scattering processes are responsible for the observed phonon-occupation redistribution during equilibration of the phonon gas after laser excitation, it is necessary to analyze the phonon-dispersion relation in the laser-excited state, which is shown in Fig. 2(a) by orange dashed lines. There are two possible third-order scattering processes which can lead to the decay of the coherent phonon. One of these processes consists of the collision (annihilation) of one $A_{1 g}$ phonon $\left(\mathbf{q}_{\mathbf{A}_{1 \mathrm{~g}}}=0\right)$ and a second phonon with momentum $\mathbf{q}$, giving rise to the creation of a third phonon with momentum q. A special case of this scattering event is the annihilation of two $A_{1 g}$ phonons and the creation of a third phonon with $\mathbf{q}=\mathbf{0}$. Energy conservation implies that the generated third phonon has a frequency equal to or larger than $v_{A_{1 g}}$. However, we do not see a significant increase in the phonon-occupation changes above $v_{A_{1 g}}$ in our simulations [see Figs. 4(d) and 4(e)]. Therefore, this process appears to play a minor role in antimony excited to an electronic temperature of $1500 \mathrm{~K}$. The other possible three-phonon collision includes the annihilation of one $A_{1 g}$ phonon and the creation of two phonons $(i, j)$ with opposite momenta, $\mathbf{q}_{\mathbf{i}}=-\mathbf{q}_{\mathbf{j}}$, as illustrated in Fig. 2(b). Notice that this process explains the population increase at $2 \mathrm{THz}$ [see Fig. 4(e)] because energy conservation implies that the two generated phonons have a frequency less than $v_{A_{1 g}}$. Additionally, many phonons around $2 \mathrm{THz}$ fulfill momentum conservation, as can be straightforwardly verified in Fig. 2(a). Note that only acoustic phonons can contribute to the increase at $2 \mathrm{THz}$, since acoustic and optical phonons are well separated in energy.

In order to determine if fourth-order phonon-phonon scattering contributes to the decay of the $A_{1 g}$ phonon at $T_{e}=1500 \mathrm{~K}$, we applied the previously detailed analysis to the three possible types of four-phonon collision processes, which can be ordered by the number of annihilated phonons, i.e., one, two, or three, with at least one $A_{1 g}$ phonon involved. We can easily neglect the fourth-order scattering processes for which three phonons are annihilated because this would lead to the creation of a phonon with a frequency above $v_{A_{1 g}}$, and such a population increase is not observed in Figs. 4(d) and 4(e). Moreover, if two $A_{1 g}$ phonons collide with each other, the highest possible frequency for one of the generated two new phonons is below $5 \mathrm{THz}$, so that the other frequency must be above $3 \mathrm{THz}$. However, such an increase of phonon occupancies is not present in Figs. 4(d) and 4(e). On the other hand, a fourth-order scattering process involving annihilation of one $A_{1 g}$ phonon and a second, different phonon of frequency $\nu_{2}$ would lead to an additional significant occupancy decrease at $v=v_{2}$ in Fig. 4. Since this is not the case, this decay process can also be excluded. Finally, the remaining fourth-order decay channel, namely, the annihilation of an $A_{1 g}$ phonon and the creation of three phonons would lead to an occupancy increase at low frequencies, which is not visible in Fig. 4. Therefore, the three-phonon decay channel represented by Fig. 2(b) is the dominating one at $T_{e}=1500 \mathrm{~K}$, consistent with the considerations from Hase et al. in Ref. [29].

In a recent study based on a model Hamiltonian and perturbation theory, it was postulated that the coherent phonon in bismuth decays through resonant squeezing [34]. Our $a b$ initio simulations on $\mathrm{Sb}$, however, show strong squeezing in most optical and acoustic phonon modes as a response to the bond softening induced by the laser pulse. From the comparison of the $A_{1 g}$-empty and full runs, we cannot definitively identify an appreciable increase of the oscillations of the mean square displacements with frequency $v_{A_{1 g}}$ due to the decay of the coherent phonon. If resonant squeezing occurs, it is a small effect compared to laser-induced squeezing (see the discussion of Fig. 3). Note that our approach is more general than that of Ref. [34] because it is valid for arbitrary ionic displacements, includes the full PES to all orders, and 576 ionic degrees of freedom.

Now we address the question of whether for increasing laser fluence more decay channels open, as intuitively expected. For this purpose, we perform the same analysis as before for an electronic temperature of $3000 \mathrm{~K}$, which corresponds to a higher-excitation density. In that case, the laser-induced bond softening is stronger and the amplitude of the coherent phonon is larger, as shown in Sec. III A. The results for the differences of the phonon-occupation numbers between the full and $A_{1 g}$ empty runs are shown in Fig. 5, averaged over the third period of the coherently excited phonon. Note that for this electronic temperature, the $v_{A_{1 g}}$ is equal to $3.37 \mathrm{THz}$.

Again one can see a clear rise of the phonon-occupation number around $v_{A_{1 g}} / 2$, which can be explained by the threephonon process described above. In contrast to the case of

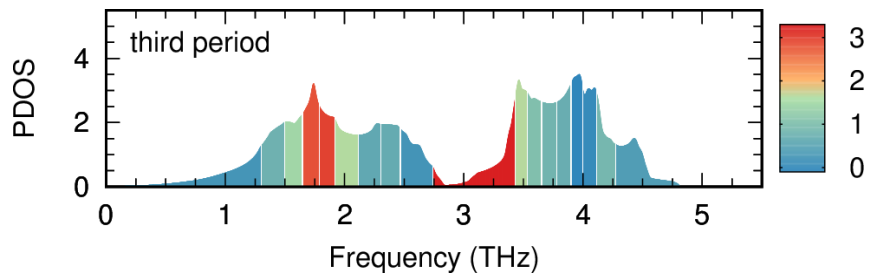

FIG. 5. Phonon density of states for an electronic temperature of $3000 \mathrm{~K}$. The difference of the phonon-occupation numbers between full and $A_{1 g}$-empty runs is shown by the colored stripes, which are summed over 96 phonon modes and averaged over the third $A_{1 g}$ period. 
$T_{e}=1500 \mathrm{~K}$, there is also a large occupancy increase near $v_{A_{1 g}}$. Another important feature of Fig. 5 is that no phonons are significantly annihilated. Keeping this in mind, the increase at $v_{A_{1 g}}$ cannot be explained by means of the third-order scattering processes discussed above. Therefore, we again analyzed the fourth-order scattering processes. We concluded that the fourth-order scattering process in which two $A_{1 g}$ phonons decay into two other phonons must be responsible for the increase of the phonon-occupation number at 3.37 $\mathrm{THz}$ because other scattering events are either energetically not possible, would lead to annihilated phonons, or generated low-frequency phonons. The identified fourth-order process, responsible for the decay of the coherent phonon at the electronic temperature $3000 \mathrm{~K}$, is illustrated in Fig. 2(c). By analyzing the phonon dispersion in Fig. 2(a), one can see that mainly optical phonons contribute to the decay of the coherent phonon. Therefore we conclude that for $T_{e}=3000 \mathrm{~K}$, both three- and four-phonon scattering processes are important for the decay of the coherent phonon.

For higher amplitudes of the coherent $A_{1 g}$ phonon, the proposed fourth-order scattering process $V^{(4)}$ should become more significant than the third-order scattering process $V^{(3)}$. This can be seen by writing both scattering processes in terms of the displacement $u_{i}$ along the phonon mode $i$,

$$
\begin{aligned}
& V^{(3)} \propto \sum_{i j} V_{i j}^{(3)} u_{A_{1 g}} u_{i} u_{j}, \\
& V^{(4)} \propto \sum_{i j} V_{i j}^{(4)} u_{A_{1 g}}^{2} u_{i} u_{j} .
\end{aligned}
$$

Therein, $V_{i j}^{(3)}$ and $V_{i j}^{(4)}$ are coupling constants. Note that $V^{(4)}$ depends quadratically on the displacement of the $A_{1 g}$ phonon and $V^{(3)}$ only linearly so that a larger amplitude leads to a more dominant influence of the fourth-order scattering processes.

In order to quantify the transition from dominant third-order processes to dominant fourth-order processes, we calculated the time evolution of the phonon energies of the full $E_{i}^{\text {full }}(t)$ and $A_{1 g}$-empty $E_{i}^{\text {empty }}(t)$ runs using Eq. (8). Then, we calculated

$$
\Delta E_{\text {third }}(t)=\sum_{i}^{v_{i} \leqslant \eta_{0}}\left[E_{i}^{\text {full }}(t)-E_{i}^{\text {empty }}(t)\right]
$$

and

$$
\Delta E_{\text {fourth }}(t)=\sum_{i}^{v_{i}>\eta_{0}}\left[E_{i}^{\text {full }}(t)-E_{i}^{\text {empty }}(t)\right],
$$

where we sum over all phonons $i$ with a phonon frequency $v_{i}$ below $\eta_{0}$ in Eq. (16) or above $\eta_{0}$ in Eq. (17). Here, $\eta_{0}=2.7$ $\mathrm{THz}$ is a qualitative "threshold" frequency with which we divide between third- and fourth-order scattering processes, respectively. Note that this classification is possible because third- and fourth-order scattering processes are well separated by frequency (see the discussion of Fig. 5). Then, we averaged over the $n^{\text {th }}-A_{1 g}$ oscillation period $(n=1,2,3, \ldots)$ and plotted the functions $\Delta E_{\text {third }}(n)$ and $\Delta E_{\text {fourth }}(n)$. We found, in all cases, a linear dependence of the form $\Delta E_{\text {third/fourth }}^{\text {it }}(n)=$ $n K_{\text {third/fourth }}+C$. The fitted constants $K_{\text {third }}$ and $K_{\text {fourth }}$ are shown in Fig. 6 as a function of the electronic temperature.

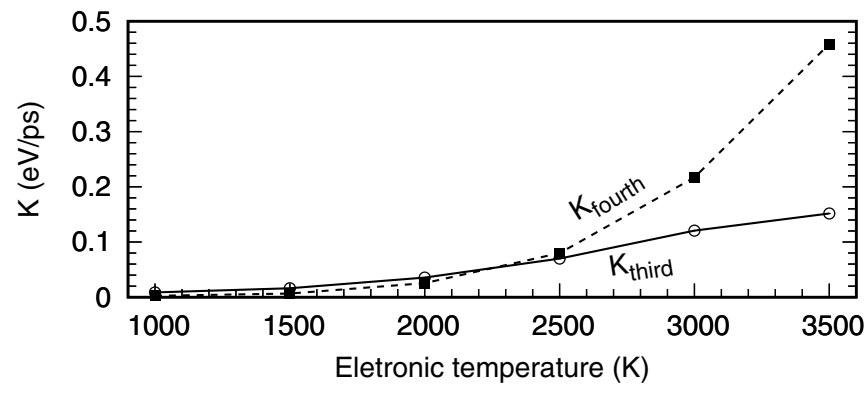

FIG. 6. Fitting parameters $K_{\text {third }}$ and $K_{\text {fourth }}$ in dependence of the electronic temperature. $K_{\text {third }}$ and $K_{\text {fourth }}$ describe the increase of the energy in the phonons interacting with the coherently excited $A_{1 g}$ phonon by third- and fourth-order scattering processes, respectively.

$K_{\text {third }}$ and $K_{\text {fourth }}$ are a qualitative measure of how fast energy is transferred from the coherently excited $A_{1 g}$ phonon mode by third- and fourth-order scattering processes, respectively. One can clearly see a crossover between dominant thirdand dominant fourth-order scattering processes at around $T_{e}=2250 \mathrm{~K}$. Such an crossover could, in principle, also be determined experimentally.

\section{CONCLUSION}

In conclusion, we have developed a nonperturbative method based on electronic-temperature-dependent, density functional, molecular dynamics simulations to describe the nonequilibration dynamics of the phonon gas in laser-excited solids. We applied the method to determine the decay channels of the coherently excited $A_{1 g}$ phonon in antimony. In this way, in contrast to usual methods, we were able to include the full dynamics of the nonequilibrium phonon gas after laser excitation. We clearly saw that the dynamics of the system is governed by the coherent phonon and by equilibration processes which occur through phonon-phonon interactions. Furthermore, our results allowed us to identify the underlying scattering processes. In particular, we found that a thirdorder scattering process, involving acoustic phonons only, is responsible for the decay at low-excitation densities. For higher laser fluences, we predicted a crossover to a fourthorder process dominating the decay. Thus, the main decay channels can, to a certain extent, be controlled by the applied laser fluence. The proposed method can be straightforwardly extended to allow a $\boldsymbol{q}$-resolved analysis.

\section{ACKNOWLEDGMENTS}

We performed our calculations at the LichtenbergHochleistungsrechner in Darmstadt and at the IT Servicezentrum Linux cluster in Kassel. We gratefully acknowledge the support from Deutsche Forschungsgemeinschaft through the Projects No. GA 465/15-2 and No. GA 465/18-1. B.B. and S.K. also gratefully acknowledge the financial support from the Otto-Braun-Fonds. 
[1] S. D. Silvestri, J. G. Fujimoto, E. P. Ippen, E. B. Gamble, L. R. Williams, and K. A. Nelson, Chem. Phys. Lett. 116, 146 (1985).

[2] T. K. Cheng, S. D. Brorson, A. S. Kazeroonian, J. S. Moodera, G. Dresselhaus, M. S. Dresselhaus, and E. P. Ippen, Appl. Phys. Lett. 57, 1004 (1990).

[3] P. Stampfli and K. H. Bennemann, Phys. Rev. B 42, 7163 (1990).

[4] V. Recoules, J. Clérouin, G. Zérah, P. M. Anglade, and S. Mazevet, Phys. Rev. Lett. 96, 055503 (2006).

[5] E. S. Zijlstra, L. L. Tatarinova, and M. E. Garcia, Phys. Rev. B 74, 220301 (2006).

[6] F. Cheenicode Kabeer, N. S. Grigoryan, E. S. Zijlstra, and M. E. Garcia, Phys. Rev. B 90, 104303 (2014).

[7] T. K. Cheng, J. Vidal, H. J. Zeiger, G. Dresselhaus, M. S. Dresselhaus, and E. P. Ippen, Appl. Phys. Lett. 59, 1923 (1991).

[8] H. J. Zeiger, J. Vidal, T. K. Cheng, E. P. Ippen, G. Dresselhaus, and M. S. Dresselhaus, Phys. Rev. B 45, 768 (1992).

[9] T. Dekorsy, H. Auer, H. J. Bakker, H. G. Roskos, and H. Kurz, Phys. Rev. B 53, 4005 (1996).

[10] T. Dekorsy, H. Kurz, X. Q. Zhou, and K. Ploog, Appl. Phys. Lett. 63, 2899 (1993).

[11] L. Min and R. J. D. Miller, Appl. Phys. Lett. 56, 524 (1990).

[12] G. C. Cho, W. Kutt, and H. Kurz, Phys. Rev. Lett. 65, 764 (1990).

[13] T. Pfeifer, T. Dekorsy, W. Kütt, and H. Kurz, Appl. Phys. A 55, 482 (1992).

[14] A. Debernardi, S. Baroni, and E. Molinari, Phys. Rev. Lett. 75, 1819 (1995).

[15] A. Debernardi, Phys. Rev. B 57, 12847 (1998).

[16] K. Ishioka, M. Kitajima, and O. V. Misochko, J. Appl. Phys. 103, 123505 (2008)

[17] H. Kumagai, I. Matsubara, J. Nakahara, and T. Mishina, arXiv:1603.00111.

[18] E. S. Zijlstra, T. Zier, B. Bauerhenne, S. Krylow, P. M. Geiger, and M. E. Garcia, Appl. Phys. A 114, 1 (2014).

[19] N. S. Grigoryan, T. Zier, M. E. Garcia, and E. S. Zijlstra, J. Opt. Soc. Am. B 31, C22 (2014).

[20] J. P. Perdew and Y. Wang, Phys. Rev. B 45, 13244 (1992).
[21] E. S. Zijlstra, N. Huntemann, A. Kalitsov, M. E. Garcia, and U. von Barth, Model. Simul. Mater. Sci. Eng. 17, 015009 (2009).

[22] S. Goedecker, M. Teter, and J. Hutter, Phys. Rev. B 54, 1703 (1996).

[23] C. Hartwigsen, S. Goedecker, and J. Hutter, Phys. Rev. B 58, 3641 (1998).

[24] We initialized the atomic positions and velocities according to Ref. [33] and then performed a 20 ps thermalization run to account for the influence of anharmonicities.

[25] The influence of the ionic temperature on the PES, calculated along the $A_{1 g}$ phonon, is approximated by

$$
F(z)=F_{0}(z)+k_{b} T \sum_{i} \ln \left[v_{i}\left(T_{e}, z\right)\right]+C .
$$

$F_{0}(z)$ is the free energy of the system at an ionic temperature $T$ of $0 \mathrm{~K}, v_{i}\left(T_{e}, z\right)$ is the frequency of the $i$ th phonon mode at an electronic temperature of $T_{e}$, and $C$ is a constant, which will be omitted.

[26] L. Waldecker, T. Vasileiadis, R. Bertoni, R. Ernstorfer, T. Zier, F. H. Valencia, M. E. Garcia, and E. S. Zijlstra, Phys. Rev. B 95, 054302 (2017).

[27] Y. Giret, A. Gellé, and B. Arnaud, Phys. Rev. Lett. 106, 155503 (2011).

[28] B. Arnaud and Y. Giret, Phys. Rev. Lett. 110, 016405 (2013).

[29] M. Hase, K. Ushida, and M. Kitajima, J. Phys. Soc. Jpn. 84, 024708 (2015).

[30] É. D. Murray, D. M. Fritz, J. K. Wahlstrand, S. Fahy, and D. A. Reis, Phys. Rev. B 72, 060301 (2005).

[31] É. D. Murray, S. Fahy, D. Prendergast, T. Ogitsu, D. M. Fritz, and D. A. Reis, Phys. Rev. B 75, 184301 (2007).

[32] A. Gambetta, C. Manzoni, E. Menna, M. Meneghetti, G. Cerullo, G. Lanzani, S. Tretiak, A. Piryatinski, A. Saxena, R. L. Martin, and A. R. Bishop, Nat. Phys. 2, 515 (2006).

[33] E. S. Zijlstra, A. Kalitsov, T. Zier, and M. E. Garcia, Phys. Rev. X 3, 011005 (2013).

[34] S. Fahy, É. D. Murray, and D. A. Reis, Phys. Rev. B 93, 134308 (2016). 